\title{
Outcome of cluster endophthalmitis in western plain region of Nepal
}

\author{
Bajimaya $\mathbf{S}^{\mathbf{1}}$, Kansakar $\mathbf{I}^{\mathbf{2}}$, Sharma $\mathrm{BR}^{3}$, Byanju $\mathbf{R}^{2}$ \\ ${ }^{1}$ General Ophthalmologist, ${ }^{2}$ Retina Clinic, ${ }^{3}$ Oculoplasty Clinic, Lumbini Eye Institute, Shree Rana-Ambika Shah Eye \\ Hospital, Bhairahawa, Nepal.
}

\begin{abstract}
Background: Post-operative endophthalmitis remains one of the most visually devastating complications of cataract surgery. Cluster endophthalmitis is defined as five or more cases of endophthalmitis occurring on a particular day in a single operating room in one centre. Excessive inflammation, particularly in the early post-operative phase, should be regarded as infective endophthalmitis. Early diagnosis and immediate intervention in such case, lead to salvage of eye as well as better visual outcome.

Objective: To find out the causative organisms and visual outcome after an outbreak of post-operative endophthalmitis in high volume cataract surgical centre.

Materials and methods: Retrospective, interventional case series of 19 patients with acute post-operative endophthalmitis after manual small incision cataract surgery in a single day, underwent vitreous tap and received intravitreal Vancomycin, amikacin and Dexamethasone. Subconjunctival vancomycin was given at the end of the procedure. Vitreous samples were stained using Grams stain, Giemsa stain and KOH mount. Samples were sent to the hospital's microbiology laboratory for culture and sensitivity testing. All patients received intravenous ciprofloxacin for 3 days and oral ciprofloxacin for 7 days. Topical Prednisolone acetate, Ofloxacin, Gentamycin and atropine were given to all patients. Patients were followed up till 6 weeks.

Results: 10 eyes had vitreous tap culture negative (52.6\%) where as 9 eyes $(47.4 \%)$ had bacterial culture growth. The culture reports showed 4 cases (21\%) of Staphylococcus epidermidis, 3 cases (15.8\%) of Staphylococcus aureus and 2 cases $(10.5 \%)$ of mixed growth. 48 hours after the intervention, 15 patients improved clinically. On sixth week follow up, 7 eyes (37\%) had visual acuity better than 6/18, 7 eyes had 6/18 to $6 / 60$ and 5 eyes had visual acuity between $1 / 60$ to $5 / 60$.

Conclusion: Intravitreal antibiotics and steroid, along with systemic ciprofloxacin and subconjunctival vancomycin has good visual outcome for post operative cluster endophthalmitis.
\end{abstract}

Key words: Manual small incision cataract surgery, Cluster Endophthalmitis, Nepal

$\mathrm{C}$ ataract extraction is the most common intraocular surgery performed worldwide. The evolution of modern cataract surgery has been characterised by a series of remarkable technical refinements. Many of the advances involved changing the type of surgical incision; that is, the transition from intracapsular cataract extraction to extracapsular cataract extraction (ECCE), followed by the transition from ECCE to sutureless manual small-incision scleral tunnel cataract surgery, and, finally, the transition of phacoemulsification from scleral tunnel incisions to clear corneal incisions. Manual small incision extra capsular cataract surgery is the most practiced surgery in developing countries. Although technical refinements in the past 2 decades have led to simplified postoperative care and faster visual recovery, post cataract surgery endophthalmitis has been reported with its incidence of $0.04 \%$ to $0.41 \%{ }^{1-6}$.
Infectious postoperative endophthalmitis is the most feared complication following ophthalmic surgery; in most cases, progression of the disease may lead to loss of vision or loss of eye as well. After aseptic precautions and sterile operating techniques, an outbreak of sightthreatening postoperative cluster endophthalmitis does rarely occur in high volume eye hospital. Early diagnosis and appropriate therapy allow the host eye to overcome the endophthalmitis causing less damage to eye. Lumbini Eye Institute (LEI) provides high level ophthalmic care to the people of western plain region of Nepal and patients from northern India. It was a second

\footnotetext{
Correspondence

Sanyam Bajimaya

General Ophthalmologist, Lumbini Eye Institute,

Shree Rana-Ambika Shah Eye Hospital, Bhairahawa-03

Rupandehi-32901, Nepal.

E-mail: sanyambajimaya@yahoo.com
} 
experience in our hospital, and the management in such situation was challenging.

\section{Materials and methods}

On $1^{\text {st }}$ November, 2007, 225 patients had under gone manual small incision extra capsular cataract extraction with polymethyl methacrylate (PMMA) Intraocular lens (IOL) implantation in Lumbini Eye Institute. Out of 225 patients, 19 eyes were suspected to have acute post-operative endophthalmitis on first post operative day. Diagnosis of endophthalmitis was made on the basis of symptoms of pain, redness and decreased visual acuity, and with signs of hypopyon and/or vitreous clouding. USG B-scan was performed in all suspected cases. With all aseptic precautions under peribulbar anaesthesia, vitreous tap was performed in the isolated minor operation room. A vitreous sample ( 0.2 to 0.3 $\mathrm{ml}$ ) was obtained by 25 gauze needle aspiration 3.5 $\mathrm{mm}$ posterior to limbus at supero-temporal pars plana area. Vitreous samples were stained using Grams stain, Giemsa stain and $\mathrm{KOH}$ mount in the operation theatre. Samples were sent to the hospital's microbiology laboratory for culture and sensitivity testing. With the immediate reports of staining and $\mathrm{KOH}$ mount, intravitreal antibiotics and steroid were administered without waiting for culture results. All eyes received intravitreal Vancomycin $(1000 \mu \mathrm{g}$ in $0.1 \mathrm{ml})$, Amikacin (400 $\mu \mathrm{g}$ in $0.1 \mathrm{ml}$ ) and Dexamethasone $(400 \mu \mathrm{g}$ in $0.1 \mathrm{ml})$. Subconjunctival Vancomycin $50 \mathrm{mg}(0.5 \mathrm{ml})$ was given at superior conjunctiva at 12 O'clock area in all cases. One drop of atropine eye drop (1\%) was instilled before pad bandage. All patients were admitted in isolate ward and were subjected to intravenous Ciprofloxacin (200mg BD) for 3 days, followed by oral ciprofloxacin $500 \mathrm{mg}$ BD for 7 days. Six hours after the intravitreal injections, topical medications Prednisolone acetate 2 hourly, commercially available Ofloxacin and Gentamycin 1 hourly, cycloplegic (atropine 1\%) 8 hourly were administered. Vitreous samples were inoculated in blood agar (aerobic and anaerobic), chocolate agar, and Brain Heart Infusion Media (BHI), all incubated at $37^{\circ}$ C. In addition, room temperature cultures on Sabouraud dextrose agar without Cycloheximide were used to grow fungi. A positive culture was defined as growth of the same organism on 2 or more media or had confluent growth on at least 1 solid medium.

Visual acuity, anterior segment biomicroscopy and fundus examination was done on the next day morning. After 48 hours, eyes with visual acuity less than hand motion with poor fundal glow had undergone core vitrectomy with repeat intravitreal vancomycin $(1000 \mu \mathrm{g}$ in $0.1 \mathrm{ml})$ and Amikacin $(400 \mu \mathrm{g}$ in $0.1 \mathrm{ml})$. Patients were followed on 2 weeks and 6 weeks post intervention. On 2 weeks follow up, uncorrected visual acuity (UCVA) and detail ocular examination was done. There after patients were asked to taper topical steroid 4 hourly for next 2 weeks and 6 hourly for next 2 weeks. Topical Ofloxacin 6 hourly and atropine 8 hourly were continued to 6 weeks. On 6 weeks follow up, all patients were subjected for uncorrected visual acuity, Pin hole Visual acuity and best corrected visual acuity (BCVA) as after refraction that was done by ophthalmic assistant. Anterior segment and posterior segment evaluation was done by general ophthalmologist and vitreo retina surgeon as well. Data were recorded and analyzed in SPSS 11.5 program.

\section{Results}

Endophthalmitis occurred in 15 right eyes (79\%) and 4 left eyes $(21 \%)$. The mean age of the 19 patients ( 7 men, 12 women) was 59 years (range 37 to 82 years). All 19 patients were non diabetic with normal level blood counts. 15 cases $(78.9 \%)$ had uneventful cataract surgery where as 1 case had difficult nucleus delivery. One eye had posterior capsule rupture (PCR) with vitreous loss and had anterior vitrectomy with posterior chamber IOL implantation. Other 2 cases had irregular scleral tunnel which required interrupted suturing (10/0 Nylon). Endophthalmitis occurred in patients who were operated by two different surgeons in separate operation rooms. Majority of patients (17/19) complained of ocular pain at early morning on first post operative day. Out of 19 patients who had acute endophthalmitis, 12 eyes $(63 \%)$ had visual acuity of hand motion (HM) and 7 eyes $(37 \%)$ had visual acuity better than $1 / 60$ but less than $6 / 60$ on first postoperative day (Table 1).

9 eyes $(47.3 \%)$ had healthy scleral tunnel wound, 9 eyes $(47.3 \%)$ had purulent discharge at wound site and 1 eye had wound gap. 15 eyes (78.9\%) had clear cornea and 4 eyes $(21.1 \%)$ had epithelial corneal oedema. Hypopion in anterior chamber (AC) was absent in 4 eyes $(21.1 \%)$. 13 eyes had an average of $1 \mathrm{~mm}$ hypopion and 2 eyes had more than $2 \mathrm{~mm}$ hypopion. 13 eyes had dense fibrinous exudates in $\mathrm{AC}$ with absent fundal glow, where as 12 eyes had vitreous clouding (63.2\%). 12 vitreous samples were clear and 7 samples were turbid.

48 hours after intravitreal antibiotics and steroid, patient's visual acuity and clinical improvement guided the need of second intervention. Pars plana (Core) vitrectomy (PPV) was performed in 2 eyes with VA PL with absent fundal glow, where as repeat intravitreal antibiotics (Vancomycin and Amikacin) was given in another 2 eyes with VA of HM (Table 1 and 2). 2 eyes had VA of counting finger (CF) with faint fundal glow. 8 eyes had VA of $1 / 60$ to $3 / 60$; whereas 5 eyes had VA $4 / 60$ to $6 / 60$. 
10 eyes $(52.7 \%)$ had vitreous sample culture negative where as 9 eyes $(47.3 \%)$ had bacterial culture growth. The culture reports showed 4 cases (21\%) of Staphylococcus epidermidis, 3 cases (15.8\%) of Staphylococcus aureus and 2 cases $(10.5 \%)$ of mixed growth. One of the polymicrobial growth was Pseudomonas aeruginosa and Staphylococcus epidermidis where as other was Pseudomonas aeruginosa and Staphylococcus aureus (Table 2).

Pattern of antibiotic sensitivity in most of the cases showed organisms sensitive to ciprofloxacin and Ofloxacin (Table 3). Among gram positive organism $100 \%(9 / 9)$ were sensitive to Ofloxacin, $89 \%(8 / 9)$ sensitive to ciprofloxacin, $89 \%(8 / 9)$ sensitive to Gentamycin, 79\% (7/9) sensitive to Tobramycin, 66\% (6/9) sensitive to Cefazoline and 55\% (5/9) sensitive to
Chloramphenicol. P. aeruginosa was the gram negative organism isolated in 2 cases of polymicrobial infection, which was resistant to Cefazoline and Chloramphenicol, but sensitive to above antibiotics.

Eyes with endophthalmitis due to Staphylococcus aureus (3 cases) had good visual outcome where as endophthalmitis due to Staphylococcus epidermidis (3 out of 5 cases) had poor visual outcome. 2 eyes with endophthalmitis due to Staphylococcus epidermidis had visual acuity of PL (10.5\%) on 48 hours of primary intervention and had undergone vitrectomy on the third post operative day. The final visual outcome was generally good. On sixth week follow up, 7 eyes (37\%) had best corrected visual acuity (BCVA) better than 6/18 and 7 eyes had BCVA 6/24 to 6/60.

Table 1: Visual acuity of patients with endophthalmitis.

\begin{tabular}{|c|c|c|c|}
\hline VA & UCVA on $1^{\text {st }}$ POD & UCVA 48 hours after $1^{\text {st }}$ intervention & BCVA on 6 weeks follow up \\
\hline PL & 0 & 2 & 0 \\
\hline HM & 12 & 2 & 0 \\
\hline $\mathrm{CF}$ & 0 & 2 & 0 \\
\hline $1 / 60-3 / 60$ & 5 & 8 & 4 \\
\hline $4 / 60-6 / 60$ & 2 & 5 & 1 \\
\hline $6 / 36-6 / 18$ & 0 & 0 & 7 \\
\hline Better than $6 / 18$ & 0 & 0 & 7 \\
\hline Total & 19 & 19 & 19 \\
\hline
\end{tabular}

Table2: Clinical characteristics, microbiology and outcome of acute endophthalimitis.

\begin{tabular}{|c|c|c|c|c|c|c|}
\hline S.No. & $\begin{array}{c}\text { Age } \\
\text { Y/Sex }\end{array}$ & $\begin{array}{c}\text { Intraoperatve } \\
\text { Complications }\end{array}$ & $\begin{array}{c}\text { VA } \\
\text { On first POD }\end{array}$ & Organisms & Repeat Intervention & $\begin{array}{c}\text { BCVA } \\
\text { 6 Weeks }\end{array}$ \\
\hline 1. & $60 / \mathrm{F}$ & Uneventful & HM & No growth & No & $6 / 18$ \\
\hline 2. & $60 / \mathrm{F}$ & $\begin{array}{c}\text { Irregular scleral } \\
\text { tunnel }\end{array}$ & $1 / 60$ & No growth & $6 / 12$ \\
\hline 3. & $66 / \mathrm{F}$ & Uneventful & $5 / 60$ & No growth & No & $6 / 6$ \\
\hline 4. & $38 / \mathrm{M}$ & Uneventful & HM & S. aureus & No & $6 / 12$ \\
\hline 5. & $65 / \mathrm{F}$ & Uneventful & HM & S. aureus & No & $6 / 24$ \\
\hline 6. & $50 / \mathrm{F}$ & $\begin{array}{c}\text { Irregular scleral } \\
\text { tunnel }\end{array}$ & $\mathrm{HM}$ & No growth & No & $3 / 60$ \\
\hline 7. & $70 / \mathrm{F}$ & Uneventful & HM & S.epidermidis & Yes, Inj. & $4 / 60$ \\
\hline 8. & $58 / \mathrm{F}$ & Uneventful & HM & No growth & No & $6 / 9$ \\
\hline 9. & $37 / \mathrm{F}$ & Uneventful & HM & S.epidermidis & Yes, Core Vitrectomy & $1 / 60$ \\
\hline 10. & $70 / \mathrm{M}$ & Uneventful & HM & S. aureus & No & $6 / 18$ \\
\hline 11. & $70 / \mathrm{M}$ & PCR & HM & No growth & Yes, Inj. & $1 / 60$ \\
\hline
\end{tabular}




\begin{tabular}{|c|c|c|c|c|c|c|}
\hline 12. & $50 / \mathrm{F}$ & Uneventful & $1 / 60$ & No growth & No & $6 / 9$ \\
\hline 13. & $65 / \mathrm{M}$ & Uneventful & $2 / 60$ & S.epidermidis & No & $6 / 12$ \\
\hline 14. & $65 / \mathrm{F}$ & Uneventful & $2 / 60$ & $\begin{array}{c}\text { P. aeruginosa } \\
\text { S.epidermidis }\end{array}$ & No & $6 / 18$ \\
\hline 15. & $50 / \mathrm{M}$ & DND & HM & $\begin{array}{c}\text { P. aeruginosa } \\
\text { S. aureus }\end{array}$ & No & $6 / 24$ \\
\hline 16. & $45 / \mathrm{M}$ & Uneventful & HM & S.epidermidis & Yes, Core Vitrectomy & $2 / 60$ \\
\hline 17. & $70 / \mathrm{F}$ & Uneventful & HM & No growth & No & $6 / 24$ \\
\hline 18. & $50 / \mathrm{F}$ & Uneventful & $2 / 60$ & No growth & No & $6 / 24$ \\
\hline 19. & $82 / \mathrm{M}$ & Uneventful & $5 / 60$ & No growth & No & $6 / 9$ \\
\hline
\end{tabular}

$\mathrm{VA}=$ Visual Acuity, BCVA=Best Corrected Visual Acuity; $\mathrm{M}=$ Male, $\mathrm{F}=$ Female, $\mathrm{PCR}=$ posterior capsule rupture, $\mathrm{DND}=$ difficult nucleus delivery, $\mathrm{POD}=$ Post operative day, Inj.= Repeat intravitreal vancomycin and amikacin, $S$ aureus=Staphylococcus aureus; $S$ epidermidis=Staphylococcus epidermidis; $P$ aeruginosa $=$ Psendomonas aeruginosa;

Table 3: Bacterial culture sensitivity reports.

\begin{tabular}{|c|c|c|c|c|}
\hline Patients & Organisms & Sensitive & Intermediate & Resistant \\
\hline Case 4. & S. aureus & $\mathrm{Cf}, \mathrm{Cz}, \mathrm{Gn}, \mathrm{Tb}, \mathrm{Nm}, \mathrm{Nx}, \mathrm{Ch}, \mathrm{Of}$ & & \\
\hline Case 5. & S. aureus & $\mathrm{Cf}, \mathrm{Gn}, \mathrm{Tb}, \mathrm{Nm}, \mathrm{Ch}, \mathrm{Nx}, \mathrm{Cz}, \mathrm{Of}$ & & \\
\hline Case 7. & S.epidermidis & $\mathrm{Cz}, \mathrm{Ch}, \mathrm{Of}, \mathrm{Nm}$ & $\mathrm{Cf}$ & $\mathrm{Nx}, \mathrm{Tb}, \mathrm{Gn}$ \\
\hline Case 9. & S.epidermidis & $\mathrm{Cf}, \mathrm{Of}, \mathrm{Nx}, \mathrm{Tb}, \mathrm{Gn}$ & $\mathrm{Ch}, \mathrm{Nm}$ & $\mathrm{Cz}$ \\
\hline Case 10. & S. aureus & Cf, ch, Gn, Tb, Of, Nx, Nm & & $\mathrm{Cz}$ \\
\hline Case 13. & S.epidermidis & $\mathrm{Cf}, \mathrm{Cz}, \mathrm{Gn}, \mathrm{Cz}$ Of, Nx, Nm & & $\mathrm{Tb}, \mathrm{Ch}$ \\
\hline \multirow{2}{*}{ Case 14.} & P. aeruginosa & $\mathrm{Cf}, \mathrm{Of}, \mathrm{Nx}, \mathrm{Tb}, \mathrm{Gn}$ & & $\mathrm{Ch}, \mathrm{Cz}$ \\
\hline & S.epidermidis & $\mathrm{Cf}, \mathrm{Of}, \mathrm{Ch}, \mathrm{Nx}, \mathrm{Tb}, \mathrm{Gn}, \mathrm{Nm}$ & & $\mathrm{Cz}$ \\
\hline \multirow[b]{2}{*}{ Case 15.} & P. aeruginosa & $\mathrm{Cf}, \mathrm{Of}, \mathrm{Nx}, \mathrm{Tb}, \mathrm{Gn}$ & \multirow{2}{*}{$\mathrm{Nm}$} & $\mathrm{Ch}, \mathrm{Cz}$ \\
\hline & S. aureus & $\mathrm{Cf}, \mathrm{Of}, \mathrm{Nx}, \mathrm{Tb}, \mathrm{Gn}, \mathrm{Nm}, \mathrm{Ch}, \mathrm{Cz}$ & & \\
\hline Case 16. & S.epidermidis & $\mathrm{Cf}, \mathrm{Of}, \mathrm{Nx}, \mathrm{Tb}, \mathrm{Gn}$ & $\mathrm{Nm}$ & $\mathrm{Cz}, \mathrm{Ch}$ \\
\hline \multicolumn{5}{|c|}{$\begin{array}{l}\text { S aureus =Staphylococcus aureus; } \text { S epidermidis=Staphylococcus epidermidis; } P \text { aeruginosa }=\text { Pseudomonas } \\
\text { aeruginosa; }\end{array}$} \\
\hline
\end{tabular}

\section{Discussion}

The purpose of this interventional case series was to document the outbreak of 19 cases of acute endophthalmitis after manual small incision extracapsular cataract surgery in single day. Our hospital, Shree Rana-Ambika Shah Eye hospital was established in 1983 and Lumbini Eye Institue (LEI) was evolved from this hospital in 2003. LEI provide high level ophthalmic care to the people of western plane region of Nepal and patients from northern India. The institute has 215 beds and serves more than 750 out patients and performs 100 to 250 different types of ocular surgeries daily. More than 30000 cataract procedures are performed annually with an average of 50 to 200 cataracts per day. In developing countries sutureless extra capsular cataract surgery with intraocular lens implantation is a safe and effective technique in areas with a high demand for cataract surgery ${ }^{7,8}$. In Nepal, the incidence of endophthalmitis after intra capsular cataract surgery (ICCE) ${ }^{9}$ has been reported to be $0.1 \%$, where as endophthalmitis after extracapsular cataract surgery $(\mathrm{ECCE})^{10}$ has been reported to be $0.3 \%$.

Our preoperative prophylaxis for cataract surgery consists of ensuring the patency of the patient's nasolacrimal duct, random blood sugar leveling, trimming of eyelashes and applying conjunctival antibiotics. We use topical Chloramphenicol drops once every 2 hours one day prior to surgery. We prescribe 
single dose of tablet ciprofloxacin $750 \mathrm{mg}$ on the day of surgery. Patients wear nonsterile hospital gowns over their own clothes, whereas feet and head are covered with clean booties and a cap, respectively. In the preoperative room, we clean the periocular skin with $10 \%$ povidone-iodine solution before giving peribulbar block. We use a reusable, sterile cotton drape to cover the surgical field. Our surgeons and assistants scrub, for 6 to 8 minutes using povidone iodine (7.5\%). Surgeons dress in sterile cotton gowns and continue to operate for 4 to 5 hours per session. We typically do not put on fresh gloves for every case. After every 5 cases, surgeons and assistants change their gloves; and in between we use $70 \%$ isopropyl alcohol. After the completion of surgery, subconjunctival injection Dexamethasone (2mg) and Gentamycin (20mg) is given superiorly covering the incision site. On the conclusion of surgery, instruments are packed in aluminum tray and then sterilised at $134^{\circ} \mathrm{C}$ and $30 \mathrm{lbs}$ of pressure using a high-speed autoclave.

Despite all the preoperative and intraoperative precautions, we report a first event of cluster endophthalmitis within our institute since last 25 years. In our case series $78.9 \%$ of endophthalmitis occurred in right eye. Our observation is similar to findings of Miller et $\mathrm{al}^{11}$ and Hani S. Al-Mezaine et $\mathrm{al}^{12}$, who report endophthalmitis cases in right eye to be $86 \%$ and $66 \%$ respectively. Most of our surgeons are righthand dominant due to which sclero-corneal incision for right eye is more temporally placed as compared to left eye. Wound leakage in a larger sutureless incision may create the potential for momentary wound incompetence. 2 eyes had irregular scleral tunnel which was apposed with interrupted sutures. The retrospective observational study on endophthalmitis at Aravind Eye Hospital $^{6}$ reports higher incidence of endophthalmitis after sutureless manual SICS $(0.12 \%)$ than after phacoemulsification $(0.03 \%)$. Kalpadakis et al. ${ }^{13}$ found a much higher incidence of endophthalmitis after largeincision ECCE (1.13\%) than after phacoemulsification $(0.57 \%)$ in a socio-economically poor community in Greece.

Out of 19 patients who had acute endophthalmitis, 12 eyes $(63 \%)$ had visual acuity of hand motion (HM) and 7 eyes (37\%) had visual acuity better than $1 / 60$ but less than $6 / 60$ on first postoperative day. Since none of the patient had visual acuity of light perception, all cases received intravitreal vancomycin, Amikacin and Dexamethasone on the basis of endophthalmitis vitrectomy study, ${ }^{14}$ but along with that we added Subconjunctival vancomycin. We also gave intravenous ciprofloxacin for 3 days followed by oral ciprofloxacin for 7 days. Chaudhry NA, et $\mathrm{al}^{15}$ has reported cluster of 10 patients with post cataract surgery endophthalmitis, for which intravitreal vancomycin and Ceftazidime along with subconjunctival injections of vancomycin (50 mg/0.5 mL), Ceftazidime $(25 \mathrm{mg} / 0.5 \mathrm{~mL})$, and Ttriamcinolone acetonide $(40 \mathrm{mg} / 0.1 \mathrm{~mL})$ were administered. 24 cases of cluster endophthalmitis have been reported from central India by Malhotra $\mathrm{S}$ et al. ${ }^{16}$ In this report, pars plana vitrectomy with intravitreal vancomycin, Amikacin and Dexamethasone was performed in all cases. Chaudhry NA, et al ${ }^{15}$ has reported 20\% (2/10, Staphylococcus epidermidis) of vitreous culture positive, where as Malhotra S et al. ${ }^{16}$ has reported 42\% (10/24, Pseudomonas aeruginosa) of culture positive. Hospital based retrospective study done in south India ${ }^{17}$ and north India ${ }^{18}$ revealed the rates of culture positive of 53\% (10/19 cases) and 38\% (47/124 cases) respectively, where as Endophthalmitis Vitrectomy Study had reported $67 \%$ culture-positive cases $^{14}$. We report $47 \%(9 / 19)$ of culture positive which includes $21 \%$ of Staph. epidermidis, $16 \%$ of Staph. aureus and $10 \%$ of mixed growth. Microbiologic reports of endophthalmitis vitrectomy study suggest that Staph. epidermidis is the single most common causative agent causing postoperative endophthalmitis ${ }^{19}$.

Innoculation of irrigating fluid, sterilised instruments, gloves and gauze pieces in blood agar, chocolate agar and brain heart infusion media revealed culture negative. To find out the theatre air contamination, culture media were kept in all operating rooms for 48 hours. Operation rooms that had cluster endophthalmitis had more than 50 colonies of bacterial growth (Staphylococcus, Diptheroids and gram negative bacilli), whereas other operating rooms had 7 to 25 colonies of bacterial growth (Staphylococcus and diptheroids). The accurate cause of the disease could be the contaminated air filter of air conditions of operation theatre. In our cases, more than $89 \%$ of organisms were sensitive to Quinolones (ciprofloxacin and Ofloxacin) and more than 79\% sensitive to aminoglycosides (Gentamycin and Tobramycin). In our report, we are not able to document the sensitivity of vancomycin and newer generation quinolones due to their unavailability. Antibiotic sensitivity pattern has not been mentioned in the reported cluster endophthalmitis from Chaudhry NA, et $\mathrm{al}^{15}$ and Malhotra S et al. ${ }^{16}$ AR Anand et al, ${ }^{20}$ has mentioned $100 \%$ gram positive organism sensitive to vancomycin, $92 \%$ sensitive to Cefazoline, $88 \%$ to Cefotaxime $88 \%$ to ciprofloxacin, $77 \%$ to Gentamycin and $73 \%$ sensitive to Ceftazidime. And among gram negative organism, $73 \%$ were sensitive to ciprofloxacin, $68 \%$ to Amikacin, $65 \%$ to Cefotaxime, $62 \%$ to Ceftazidime and $55 \%$ were sensitive to Gentamycin.

On 48 hours after the first intervention, 2 cases deteriorate their visual acuity to light perception which had undergone pars plana vitrectomy. On 6 weeks follow up, 7 eyes (37\%) had best corrected visual acuity 
(BCVA) better than 6/18 and 7 eyes had BCVA 6/24 to $6 / 60.2$ out of 10 eyes $(20 \%)$ with culture negative cases had poor visual outcome (VA 1/60 to 3/60), where as 3 out of 9 eyes (33\%) with culture positive had poor visual outcome (VA 1/60 to 4/60). Staphylococcus epidermidis (3 out of 9 eyes) remained as the most virulent organism causing poor visual outcome among culture positive cases. None of the eyes underwent evisceration. Our results show that a positive culture and poor initial visual acuity are risk factors for decreased final visual acuity which is in agreement with endophthalmitis vitrectomy study ${ }^{14}$. The EVS study recommends no difference in final visual acuity or media clarity with or without the use of systemic antibiotics. The retrospective study done in Turkey ${ }^{21}$ has used intravenous vancomycin and Ceftazidime for severe cases of acute onset endophthalmitis after cataract surgery and oral ciprofloxacin for less severe cases. They found no difference in Systemic ciprofloxacin in the visual outcome among patients who received oral antibiotics, intravenous therapy, or no systemic therapy. In our case series we did not have control group to compare the role of intravenous ciprofloxacin. Our current case series is also limited by not having vancomycin and Amikacin sensitivity test report. We did follow the guidelines of EVS study, but our follow up period was very short which could be due to very low socio economical condition of patients. About $74 \%$ $(14 / 19)$ of our patient had achieved best corrected visual acuity (BCVA) better than or equal to 6/60. Z I carrim et $\mathrm{al}^{22}$ and BOSU survey ${ }^{23}$ have reported final BCVA better than or equal to $6 / 60$ in $80 \%$ and $66 \%$ respectively. Good visual outcomes in 14 cases of our series must be due to early presentation as patients were hospitalized on the day of surgery, second factor must be due to abrupt intervention, and third factor due to our antibiotic protocol. Poor visual outcome in our case series could be due to posterior capsule rupture, culture negative and poor vision at first post operative day. One could add slit lamp examination of all patients on first post operative day, which is usually not done in high volume cataract surgery hospital. We conclude intravitreal vancomycin, Amikacin and Dexamethasone along with subconjunctival vancomycin has good anatomical and functional outcome in cluster endophthalmitis.

\section{Conclusion}

Intravitreal antibiotics and steroid, along with systemic ciprofloxacin and subconjunctival vancomycin has good visual outcome for cluster endophthalmitis after manual small incision extracapsular cataract extraction. Eyes with endophthalmitis due to Staphylococcus aureus had good visual outcome where as endophthalmitis due to Staphylococcus epidermidis had poor visual outcome.

\section{References}

1. Miller JJ, Scott IU, FlynnHWJr, Smiddy WE, Newton J, MillerD. Acute-onset endophthalmitis after cataract surgery (2000-2004): incidence, clinical settings, and visual acuity outcomes after treatment. Am J Ophthalmol. 2005; 139:983-7.

2. Bell CM, Hatch WV, Cernat G, Urbach DR. Surgeon volumes and selected patient outcomes in cataract surgery. Ophthalmology. 2007; 114:405-10.

3. Javitt JC, Vitale S, Canner JK, et al. National outcomes of cataract extraction; endophthalmitis following inpatient surgery. Arch Ophthalmol. 1991; 109:1085-9.

4. Kresloff MS, Castellarin AA, Zarbin MA. Endophthalmitis. Surv Ophthalmol. 1998; 43:193-224.

5. Schmitz S, Dick HB, Krummenauer F, Pfeiffer N. Endophthalmitis in cataract surgery; results of a German survey. Ophthalmology. 1999; 106:1869-77.

6. Ravindran RD, Venkatesh $\mathrm{R}$, Chang DF, Sengupta S, Gyatsho J, Talwar B. Incidence of post-cataract endophthalmitis at Aravind Eye Hospital. J Cataract Refract Surg 2009; 35:629636

7. A Hennig, J Kumar, D Yorston and A Foster. Sutureless cataract surgery with nucleus extraction: outcome of a prospective study in Nepal. Br. J. Ophthalmol. 2003;87;266-70.

8. Ruit S, Paudyal G, Gurung R, et al. An innovation in developing world cataract surgery: sutureless extracapsular cataract extraction with intraocular lens implantation. Clin Exp Ophthalmol. 2000;28:274-9.

9. Snellingen T, Shrestha JK, Husain R, Rao GN, et al. The South Asian Cataract Management Study: Complications, Vision Outcomes, and Corneal Endothelial Cell Loss in a Randomized Multicenter Clinical Trial Comparing Intracapsular Cataract Extraction with and without Anterior Chamber Intraocular Lens Implantation. Ophthalmology.2000;107(2):23140.

10. Ruit S, Robin AL, Pokhrel RP, Sharma A, DeFaller J, Maguire PT. Long-term results of extracapsular cataract extraction and posterior chamber intraocular lens insertion in Nepal. Trans Am Ophthalmol Soc. 1991;89:59-72.

11. Miller JJ, Scott IU, Flynn HW Jr, Smiddy WE, Newton J, Miller D. Acute-onset endophthalmitis after cataract surgery (2000-2004): incidence, 
clinical settings, and visual acuity outcomes after treatment. Am J Ophthalmol. 2005; 139:983- 7 .

12. Hani S. Al-Mezaine, Kangave D, Al-Assiri A, Al-Rajhi AA. Acute-onset nosocomial endophthalmitis aftercataractsurgery. Incidence, clinical features, causative organisms, and visual outcomes. J Cataract Refract Surg. 2009; 35:643-9.

13. Kalpadakis P, Tsinopoulos I, Rudolph G, Schebitz K, Froehlich SJ. A comparison of endophthalmitis after phacoemulsification or extracapsular cataract extraction in a socio-economically deprived environment: a retrospective analysis of 2446 patients. Eur J Ophthalmol. 2002; 12:395-400.

14. Endophthalmitis Vitrectomy Study Group. Results oftheEndophthalmitis VitrectomyStudy; a randomized trial of immediate vitrectomy and of intravenous antibiotics for the treatment of postoperative bacterial endophthalmitis. Arch Ophthalmol. 1995; 113:1479-96.

15. Chaudhry NA, Lavaque AJ, Scott IU, Flynn HW Jr., Liggett PE. A Cluster of Patients with Acute-Onset Endophthalmitis Following Cataract Surgery. Ophthalmic Surg Lasers Imaging. 2005;36:205-10.

16. Malhotra S, Mandal P, Patanker G, Agrawal D. Clinical profile and visual outcome in cluster endophthalmitis following cataract surgery in Central India. Indian J Ophthalmol. 2008; 56(2):157-8.
17. Lalitha P, Rajagopalan J, Prakash K, Ramasamy K, Prajna NV, Srinivasan M. Postcataract Endophthalmitis in South India, Incidence and Outcome. Ophthalmology. 2005;112:1885-90.

18. Gupta A, Gupta V, Gupta A, et al. Spectrum and clinical profile of post cataract surgery endophthalmitis in North India. Indian J Ophthalmol. 2003;51:139-45.

19. Han DP, Wisniewski SR, Wilson LA, Barza $\mathrm{M}$, Vine AK, Doft BH et al. Spectrum and susceptibilities of microbiologic isolates in the endophthalmitis vitrectomy study. Am J Ophthalmol. 1996;122:1-17.

20. Anand AR, Therese KL, Madhavan HN. Spectrum of aetiological agents of postoperative endophthalmitis and antibiotic susceptibility of bacterial isolates. Indian $\mathrm{J}$ Ophthalmol. 2000;48(2):123-8.

21. Altan $\mathrm{T}$, Acar $\mathrm{N}$, et al. Acute-onset endophthalmitis after cataract surgery. Success of initial therapy, visual outcomes, and related factors. Retina. 2009;29:606-12.

22. Z I carrim, J Richardson, WN Wykes. Incidence and visual outcome of acute endophthalmitis after cataract surgery- the experience of an eye department in Scotland. Br. J. Ophthalmol. 2009;93;721-5.

23. Kamalarajah S, Silvestri G, Sharma N, et al. Surveillance of endophthalmitis following cataract surgery in the UK. Eye 2004;18:5807. 